\title{
The Triple-Flash Illusion Reveals a Driving Role of Alpha-Band Reverberations in Visual Perception
}

\author{
(DRasa Gulbinaite, ${ }^{1,2}$ Barkın İlhan, ${ }^{3}$ and $\oplus^{\circ}$ Rufin VanRullen ${ }^{1,2}$ \\ ${ }^{1}$ Centre National de la Recherche Scientifique, Unité Mixte de Recherche 5549, Faculté de Médecine Purpan, Toulouse, 31000 France, ${ }^{2}$ Université de \\ Toulouse, Centre de Recherche Cerveau et Cognition, Université Paul Sabatier, Toulouse, 31052 France, and ${ }^{3}$ Meram Medical Faculty, Konya NE University, \\ Konya, 42080 Turkey
}

The modulatory role of spontaneous brain oscillations on perception of threshold-level stimuli is well established. Here, we provide evidence that alpha-band $(\sim 10 \mathrm{~Hz})$ oscillations not only modulate perception of threshold-level sensory inputs but also can drive perception and generate percepts without a physical stimulus being present. We used the "triple-flash" illusion: Occasional perception of three flashes when only two spatially coincident veridical ones, separated by $\sim 100 \mathrm{~ms}$, are presented. The illusion was proposed to result from superposition of two hypothetical oscillatory impulse response functions generated in response to each flash: When the delay between flashes matches the period of the oscillation, the superposition enhances a later part of the oscillation that is normally damped; when this enhancement crosses perceptual threshold, a third flash is erroneously perceived (Bowen, 1989). In Experiment 1, we varied stimulus onset asynchrony and validated Bowen's theory: The optimal stimulus onset asynchrony for illusion to occur was correlated, across human subjects (both genders), with the subject-specific impulse response function period determined from a separate EEG experiment. Experiment 2 revealed that prestimulus parietal, but no occipital, alpha EEG phase and power, as well as poststimulus alpha phase-locking, together determine the occurrence of the illusion on a trial-by-trial basis. Thus, oscillatory reverberations create something out of nothing: A third flash where there are only two.

Key words: alpha oscillations; EEG; individual alpha peak frequency; phase; power; triple-flash illusion

Significance Statement

We highlight a novel property of alpha-band $(\sim 10 \mathrm{~Hz})$ oscillations based on three experiments (two EEG and one psychophysics) by demonstrating that alpha-band oscillations do not merely modulate perception, but can also drive perception. We show that human participants report seeing a third flash when only two are presented (the "triple-flash" illusion) most often when the interflash delay matches the period of participant's oscillatory impulse response function reverberating in alpha. Within-subject, the phase and power of ongoing parietal, but not occipital, alpha-band oscillations at the time of the first flash determine illusory percept on a trial-by-trial basis. We revealed a physiologically plausible mechanism that validates and extends the original theoretical account of the triple-flash illusion proposed by Bowen in 1989.

\section{Introduction}

Spontaneous rhythmic fluctuations in various frequency bands have been consistently reported to affect perception across several sensory modalities (VanRullen, 2016b). Most studies found modulatory effects of prestimulus alpha-band $(\sim 10 \mathrm{~Hz})$ power, phase, and frequency on threshold-level stimulus detection, per-

\footnotetext{
Received Dec. 23, 2016; revised June 8, 2017; accepted June 17, 2017.

Author contributions: R.G., B.I., and R.V. designed research; R.G. and B.I performed research; R.G. contributed unpublished reagents/analytic tools; R.G. analyzed data; R.G., B.I, and R.V. wrote the paper.

This work was supported by the European Research Council Grant P-CYCLES 614244 to R.V. We thank Mike X. Cohen for advice on data analysis.

The authors declare no competing financial interests.

Correspondence should be addressed to Dr. Rasa Gulbinaite, Centre de Recherche Cerveau et Cognition, CNRS, UMR 5549, BP 25202, 31052 Toulouse Cedex, France. E-mail: rasa.gulbinaite@gmail.com.

DOI:10.1523/JNEUROSCI.3929-16.2017

Copyright $\odot 2017$ the authors $\quad 0270-6474 / 17 / 377219-12 \$ 15.00 / 0$
}

ceptual and temporal discrimination (Busch et al., 2009; Roberts et al., 2014; Baumgarten et al., 2015; Samaha and Postle, 2015). These perceptual consequences of endogenous oscillations imply that perception is inherently rhythmic and operates in a form of perceptual cycles, with periods of high and low excitability (Dugué et al., 2011; VanRullen et al., 2011). However, most evidence for rhythmicity in perception is based on modulatory effects of ongoing oscillations on stimulus processing. For example, oscillatory phase at stimulus onset modulates stimulus visibility by 10\%-15\% (Mathewson et al., 2009; Busch and VanRullen, 2010; Dugué et al., 2011). Do brain oscillations merely modulate perception, or can they also, under certain conditions, drive perception over perceptual threshold and generate a percept without a physical stimulus?

In the "triple-flash" visual illusion, two brief light pulses separated by $\sim 100 \mathrm{~ms}$ are sometimes perceived as three (Bowen, 
1989). The effect was theoretically explained by the superposition of two damped oscillatory impulse response functions (IRF) generated in response to each stimulus flash. The third illusory flash is perceived when the delay between veridical flashes matches the period of oscillatory IRF. In this case, the later part of the oscillation is enhanced; and when this enhancement crosses perceptual threshold, a third illusory flash is perceived (see Fig. $1 B$, middle). However, when the delay does not match the period, the later part of the oscillation is dampened, and only two flashes are perceived (see Fig. $1 B$, top, bottom). Thus, Bowen's theoretical account of the triple-flash illusion assumes that the brain response to a single flash of light is oscillatory.

Empirical tests of Bowen's insightful predictions, and the role of oscillations in the triple-flash illusion, have not yet been demonstrated. Several findings, however, indicate that alpha-band oscillations could be involved in the generation of illusory third-flash percepts. First, the optimal two-flash delay of $\sim 100$ ms corresponds to the average period of alpha-band oscillations. Additionally, the optimal interflash delay for the illusion to be perceived varies across individuals (Bowen, 1989), and so does the alpha peak frequency across individuals (individual alpha peak frequency, IAF) and across brain areas within individual (Doppelmayr et al., 1998; Haegens et al., 2014). Second, variations in occipital IAF are causally linked to the temporal properties of visual perception, such that faster occipital alpha oscillations are associated with finer temporal resolution in perception (Cecere et al., 2015; Samaha and Postle, 2015). Third, there is empirical evidence that the response to a single flash is indeed oscillatory and reverberates at $\sim 10 \mathrm{~Hz}$ (VanRullen and Macdonald, 2012).

The triple-flash illusion appears similar to other phantom-flash illusions (Apthorp et al., 2013), for example, the sound-induced double-flash illusion, whose temporal window is causally related to alpha-band oscillations (Cecere et al., 2015). However, the "tripleflash" illusion is purely endogenous, whereas perception of other phantom-flash illusions requires simultaneous presentation of additional stimuli, either in a different modality or in a different spatial location.

Understanding the role of oscillations in the triple-flash illusion is critical to the idea of perceptual cycles, as the illusory third-flash is potentially caused by perceptual reverberations. According to this line of reasoning, cortical excitability and stimulus-evoked responses determined by the power and phase of ongoing alpha-band oscillations at the moment of the first flash could have carryover effects for several alpha cycles (Remond and Lesevre, 1967; Jansen and Brandt, 1991; Busch et al., 2009; Mathewson et al., 2009; Fiebelkorn et al., 2013). In Experiment 1 , we directly tested whether illusory third-flash perception could result from summed reverberations of visual responses as proposed by Bowen (1989). In Experiment 2, we investigated prestimulus and stimulus-related effects of alpha phase, power, and frequency (separately for occipital and parietal alpha sources) on the illusory third-flash perception.

\section{Materials and Methods}

\section{Experiment 1}

This experiment consisted of two parts. In the first part, we estimated subject-specific optimal stimulus onset asynchrony ( $\mathrm{SOA}$ ) of the tripleflash illusion using a psychophysical approach. In the second part, we established the subject-specific IRF using EEG recordings obtained during white noise flicker stimulation.

Participants. Thirty participants ( 12 females, mean age 27.4 years) took part in the psychophysics experiment. All participants had normal or corrected-to-normal vision. The data from 3 participants were excluded from the analyses: 2 due to low accuracy on easy-to-discriminate long
SOA three-flash trials and 1 due to bias toward reporting illusions on very short SOA trials, with no discernable preferred SOA for the triple-flash illusion to be perceived. The study was conducted in accordance with the Declaration of Helsinki and approved by the local ethics committee. Written informed consent was obtained from all participants before the experiment.

Stimuli and procedure. Stimuli for the triple-flash experiment were two or three uniform white circles (radius $3.5^{\circ}$ ) presented on a black background in rapid succession peripherally above the fixation dot (eccentricity $7^{\circ}$ visual angle). The viewing distance was constrained by the chin rest positioned $57 \mathrm{~cm}$ away from a 17 inch CRT monitor $(600 \times 480$ pixels; $160 \mathrm{~Hz}$ vertical refresh rate). On each trial, either two or three circles were presented for a duration of one screen refresh $(6.25 \mathrm{~ms})$ with variable SOA (Fig. 1A). After the offset of the last flash, the screen remained black until a response was made. Following the response, the next trial started after a variable delay (1000-1500 ms). For a majority of participants $(N=20)$, the SOA on two-flash trials was randomly selected from 1 of 10 possible SOAs (50, 62.5, 75, 87.5, 100, 112.5, 125, 137.5, 150, $175 \mathrm{~ms}$ ), with 80 trials per SOA; and on three-flash trials from 8 possible SOAs $(25,31.25,37.5,50,75,87.5,175$, and $250 \mathrm{~ms})$, with 50 trials per SOA. For the remaining 10 participants, a finer sampling of SOAs was used: for the two-flash trials, 17 SOAs ( 50 to $250 \mathrm{~ms}$, in steps of $12.5 \mathrm{~ms}$ ), with 40 trials per SOA; and for the three-flash trials, 27 SOAs (25 to 125 $\mathrm{ms}$, in steps of $6.25 \mathrm{~ms}$, and 125 to $250 \mathrm{~ms}$, in steps of $12.5 \mathrm{~ms}$ ), with 20 trials per SOA. The overall probability of three flash trials was $33 \%$ (coarsely sampled SOAs) or $44 \%$ (finely sampled SOAs) throughout the experiment. The task consisted of 20 practice trials and 1200 (coarsely sampled SOAs) or 1220 (finely sampled SOAs) experimental trials separated in blocks of 100 trials. Participants were tested in a dark room. They were instructed to keep their eyes on the fixation dot throughout the experiment, and to make speeded responses without sacrificing accuracy. "Left arrow" and "right arrow" keys were used to indicate perception of two and three flashes, respectively. Participants were encouraged to take breaks after each block. The experiment lasted $\sim 1 \mathrm{~h}$.

In a separate experimental session, we recorded EEG while participants monitored a peripheral disk stimulus, whose luminance randomly varied every screen refresh with the constraint that the power spectrum of the luminance time course was flat between 0 and $80 \mathrm{~Hz}$ (i.e., white noise). Stimulus size, position, viewing distance, background, and screen refresh rate were kept identical to the triple-flash experimental session. Participants were instructed to detect target stimuli (a small circle surrounded by a darker annulus) presented in the center of the flickering stimulus by pressing a button. There were $2-4$ targets presented during each 6.25-s-long trial. Target stimulus detectability (set to 50\% using adaptive staircase procedure based on the performance during the first 30 trials) was manipulated by changing the relative contrast between the small circle and the annulus. The beginning of each trial was self-paced using a button press. The experiment lasted $\sim 1 \mathrm{~h}$ and was divided in 8 blocks, with 48 trials in each block. Participants were encouraged to take rest breaks after each block. Further details on the rationale of this EEG study and additional analyses can be found in Brüers and VanRullen (2017).

Data analysis. To evaluate whether the triple-flash perception is related to oscillatory IRF, we correlated the period of subject-specific IRF with the subject-specific optimal SOA for illusory perception. Robustness of correlations was assessed by performing a bootstrapping procedure (resampling with replacement) using the Robust Correlation Toolbox (Pernet et al., 2012).

The period of subject-specific IRF was obtained by performing the following analysis steps. First, EEG data were preprocessed using the same pipeline as for the data in the Experiment 2 (see below), with a few exceptions: (1) data were downsampled to $160 \mathrm{~Hz}$ to match the rate of stimulus luminance changes; (2) epochs were -250 to $6500 \mathrm{~ms}$ relative to the onset of the luminance sequence; and (3) trials containing eye blink artifacts during luminance sequences were excluded from the analyses. Second, single-trial EEG time-series were cross-correlated with stimulus luminance time series at lags between -400 and $1300 \mathrm{~ms}$ in steps of 1/160. Third, the fast Fourier transform of the trial-average crosscorrelation result in the -400 to $1300 \mathrm{~ms}$ was performed. The data were zero-padded to obtain $0.1 \mathrm{~Hz}$ frequency resolution. The period of 
A

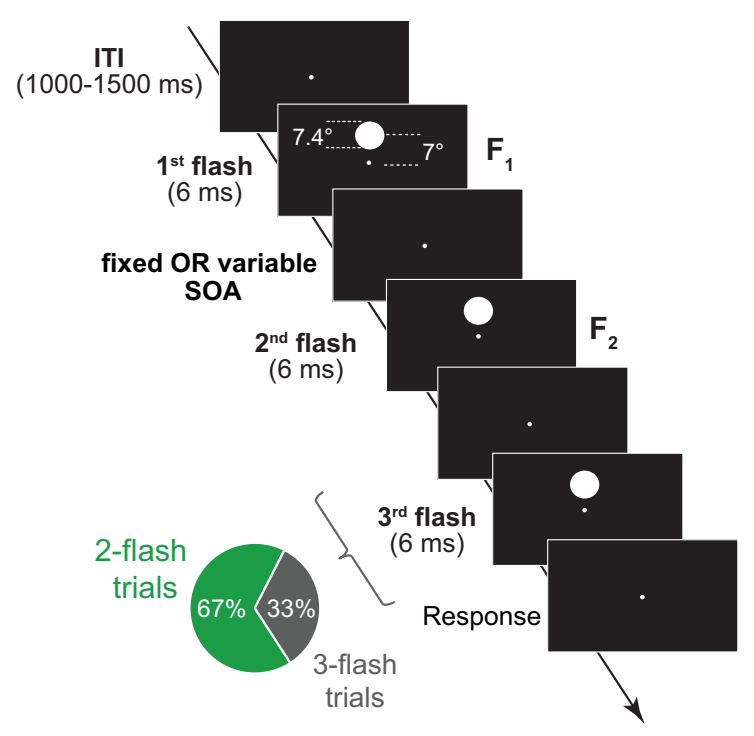

B
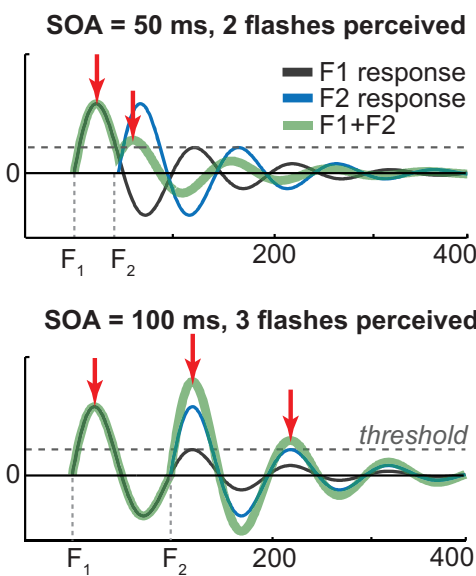

$\mathrm{SOA}=150 \mathrm{~ms}, 2$ flashes perceived

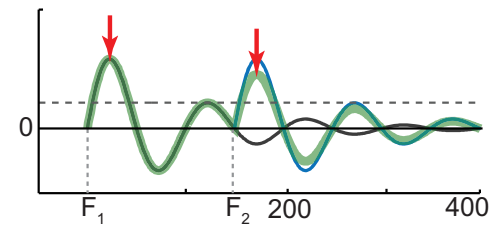

Figure 1. Trial structure and Bowen's theoretical model. $A$, On each trial, two or three high-contrast circle stimuli were presented in rapid succession above the fixation dot. SOA on two-flash trials was either variable (Experiment 1) or fixed (Experiment 2); three-flash trial SOA was variable in both experiments. B, Oscillatory model of brain response to two consecutive light flashes (adapted from Bowen 1989). Thin black and blue lines indicate an IRF to the first and the second flash, respectively. Thick green line indicates a linear sum of IRFs to two flashes. Horizontal dashed lines indicate a hypothetical perceptual threshold which, when exceeded, gives rise to the perception of a flash. Top and Bottom, The delay between veridical flashes is suboptimal for the third-flash perception to occur (i.e., too short, $S 0 \mathrm{~A}=50 \mathrm{~ms}$, and too long SOA $=150 \mathrm{~ms}$ ). Middle, Scenario when the second flash is presented $\sim 100 \mathrm{~ms}$ relative to the first flash, resulting in a perfect superposition of the two IRFs and a third-flash percept.

subject-specific IRF was determined by finding the peak frequency $(f)$ in the range of $6-14 \mathrm{~Hz}$, and expressing it as period in milliseconds $(1 / f)$.

Subject-specific optimal SOA for the triple-flash illusion to be perceived was determined by fitting symmetrical and nonsymmetrical functions to the behavioral performance on two-flash trials (proportion of two-flash trials perceived as three). Initial to-be-fitted model parameters were based on each subject's empirical data and used as an input for fminsearch Matlab function. We used Gaussian (1), Weibull (2), and ex-Gaussian (3) functions as follows:

$$
f(x)=a e^{-\frac{(x-b)^{2}}{2 c^{2}}}
$$

where $a$ is Gaussian peak $y$-axis value (initially set to maximum number of illusions perceived by participant), $b$ is Gausian peak $x$-axis value (initially set to SOA of maximum number of illusions), and $c$ is width of the Gaussian (initially set to $0.05 \mathrm{~s}$ ) as follows:

$$
f(x)=c\left(\frac{x}{a}\right)^{b-1} e^{-\left(\frac{x}{a}\right)^{b}}
$$

where $a$ is the $x$-axis scaling factor (initially set to SOA of maximum number of illusions perceived by participant), $b$ defines the shape of the curve (initially set to 4 ), and $c$ scales the curve along the $y$-axis (initially set to twice the maximum number of illusions).

$$
f(x)=h \frac{\lambda}{2} e^{\frac{\lambda}{2}\left(2 \mu+\lambda \sigma^{2}-2 x\right)} \operatorname{erfc}\left(\frac{\mu+\lambda \sigma^{2}-x}{\sqrt{2} \sigma}\right)
$$

where $\lambda=1 / \tau$, and $\tau$ is an exponential decay parameter (initially set to 0.1 ), $\mu$ is mean of the Gaussian (initially set to half the SOA with maximum number of illusions), $\sigma$ is variance of the Gaussian (initially set to $0.05 \mathrm{~s}$ ), and $h$ is the $y$-axis scaling factor (initially set to maximum number of illusions divided by 4 ).

Goodness of fit at each fminsearch iteration and across the three different fitting functions was evaluated using $R^{2}$, which is the amount of variance accounted for.

\section{Experiment 2}

The purpose of Experiment 2 was to characterize EEG changes that accompany perception of the third-flash illusion relative to no-illusion trials with physically identical stimuli.

Participants. EEG data were recorded from 35 participants ( 18 females, mean age 26.5 years); 18 of them also participated in Experiment 1. All participants had normal or corrected-to-normal vision. The study was conducted in accordance with the Declaration of Helsinki and approved by the local ethics committee. Written informed consent was obtained from all participants before the experiment.

Stimuli and procedure. Compared with the procedure of Experiment 1, two-flash trial SOA was fixed at $87.5 \mathrm{~ms}$. The choice of this SOA was based on the results of Experiment 1, where maximal number of illusions on average across participants based on ex-Gaussian fits was $92 \mathrm{~ms}$ ( $\mathrm{SD}=$ $17 \mathrm{~ms})$. As in Experiment 1, three-flash trial SOA was variable (25, 31.25, $37.5,50,75,87.5,175$, and $250 \mathrm{~ms}$ ), and overall proportion of three-flash trials was kept at $33 \%$ throughout the experiment. To avoid muscle artifacts in EEG, viewing distance was unconstrained by the chin rest but still kept at $\sim 57 \mathrm{~cm}$.

Data acquisition and preprocessing. EEG data were recorded using 64channel ActiveTwo BioSemi system (for detailed description of the hardware, see www.biosemi.com) at $1024 \mathrm{~Hz}$ sampling rate. Offline, the data were downsampled to $512 \mathrm{~Hz}$ and rereferenced to the average activity over all electrodes. Continuous EEG recordings were bandpass filtered at $0.5-80 \mathrm{~Hz}$, and electrical line noise was removed using a notch filter (band-stop 47-53 Hz). The data were then epoched (-1500 to $2000 \mathrm{~ms}$ relative to the first stimulus onset) and baseline-corrected with respect to the time window of -200 to $0 \mathrm{~ms}$ relative to the first stimulus onset.

Artifact removal was done in two steps. First, the data were visually inspected and trials containing muscle artifacts or eye blinks during and $800 \mathrm{~ms}$ before the stimulus presentation were removed. The second artifact rejection step involved independent components analysis (ICA) (Delorme and Makeig, 2004). Components that did not account for any brain activity, such as eye movements or noise, were subtracted from the data (on average, 1.4 components per subject). Furthermore, trials with 
reaction time of 3 SDs longer than subject's mean reaction time were excluded from the analysis. On average, $90.6 \%$ of two-flash trials per subject were included in the analysis $(\mathrm{SD}=4.4 \%)$.

Alpha-band source separation. To account for variability in alpha peak frequency across individuals, and across brain regions within an individual (Haegens et al., 2014), we separated parietal and occipital alpha sources based on ICA using JADE algorithm as implemented in EEGLAB and dipole fitting using DIPFIT plug-in of the EEGLAB toolbox (Oostenveld and Oostendorp, 2002; Delorme and Makeig, 2004). First, eyeblink and movement artifact-free data were bandpass filtered at 5-15 Hz. The filter kernel was created using Matlab firls function with a filter order of 307 ( $3 \times$ (sampling rate/lower bound of the filter), and 15\% transition zones (Cohen, 2014a). Thereafter, the ICA was performed on temporally filtered data using only the prestimulus time window $(-1000$ to $0 \mathrm{~ms}$, where 0 is the first stimulus onset), obtaining 20 independent components (ICs). For each IC, a single-equivalent current dipole model was fitted using three-layer BEM template model based on the standard MNI's brain template from the DIPFIT plug-in. One parietal and one occipital IC were selected based on the spatial proximity of the fitted dipoles to the reference anatomical coordinates, with constraints that selected equivalent dipoles had $<15 \%$ residual variance from the spherical forward-model scalp projection, and were located inside the model brain volume. Reference anatomical coordinates for parietal regions of interest (ROIs) were centered on the left and on the right Brodmann area 7 (right-side MNI coordinates: -20 , -90 , 0; left-side MNI coordinates: 20, -90, 0); for occipital ROIs, mean coordinates of Brodmann areas 17 and 18 were used (right-side MNI coordinates: $-20,-70$, 50; left-side MNI coordinates: 20,-70, 50) (Haegens et al., 2014).

IAF at occipital and parietal alpha sources was estimated by taking the fast Fourier transform of single-trial IC time series in the -1000 to $0 \mathrm{~ms}$ window. The data were zero-padded to obtain $0.1 \mathrm{~Hz}$ frequency resolution. The absolute value of fast Fourier transform coefficients was squared and averaged across trials. The IAF was determined as the peak in the range of 6-14 Hz. This frequency window was chosen based on a large-sample study $(N=96)$ by Bazanova and Vernon (2014), which demonstrated that individual alpha peak can vary from 6 to $14 \mathrm{~Hz}$. To make sure that IAF estimation using all trials was independent from the magnitude of alpha-band power, we conducted a control analysis to test for differences in IAF on high versus low alpha-band power trials. Highand low-alpha power trials were defined by means of a median split of all trials with respect to alpha power (mean in the $10 \pm 3 \mathrm{~Hz}$ frequency window) during prestimulus interval. Of the total of 33 participants on low alpha power trials, the parietal IAF could be determined for 30 , and the occipital IAF for 28 participants (IAF was determined using the same procedure as described above). There were no statistically significant differences for IAF on high versus low alpha-band power trials either for parietal $\left(t_{(29)}=0.136, p=0.892\right)$ or occipital alpha sources $\left(t_{(27)}=\right.$ $0.431, p=0.670)$. Given this result, and the fact that IAF determined from all trials has higher signal-to-noise ratio and thus provides a more reliable estimate of IAF, for between-subject analyses we used IAF defined from all trials.

Independent component time-frequency analyses. Epoched unfiltered data were multiplied by ICA unmixing matrix from selected subjectspecific parietal and occipital components to obtain component singletrial time series. Time-frequency decomposition was performed by convolving single-trial data from parietal and occipital ICs with complex Morlet wavelets, defined as follows:

$$
e^{i 2 \pi f_{i} t} e^{-t^{2} /\left(2 \sigma^{2}\right)}
$$

where $t$ is time, $f_{i}$ is frequency which ranged from 2 to $40 \mathrm{~Hz}$ in 39 logarithmically spaced steps, and $\sigma$ is the width of each frequency band defined as $n /\left(2 \pi f_{i}\right)$, where $n$ is a number of wavelet cycles that varied from 1 to 7 in logarithmically spaced steps to obtain comparable frequency precision at low and high frequencies.

Instantaneous power was computed as the square of the analytic signal $\mathrm{Z}$ (a complex result of convolution) and averaged across trials (i.e., power $=$ $\operatorname{Re}[Z(t)]^{2}+\operatorname{Im}[Z(t)]^{2}$ ). Thus, obtained power values were then baselinecorrected by converting to decibel scale $\left(10 \log _{10}\right.$ (power/baseline $\left.)\right)$, where condition-average power from -400 to $-100 \mathrm{~ms}$ prestimulus period served as the baseline. Condition-average rather than condition-specific baseline was used to avoid introducing spurious power differences in the poststimulus period.

To evaluate the effects of alpha phase on illusory third-flash perception, we compared phase distributions on illusion versus nonillusion two-flash trials using phase opposition sum (POS), which is expressed as a function of intertrial phase clustering (ITPC) of illusion and nonillusion trials relative to ITPC of all trials (VanRullen, 2016a) as follows:

$$
\mathrm{POS}=\mathrm{ITPC}_{\text {illusion_trials }}+\mathrm{ITPC}_{\text {non-illusion trials }}-2 \mathrm{ITPC}_{\text {All trials }}
$$

where ITPC $=\left|\frac{1}{n} \sum_{j=1}^{n} e^{i \varphi_{t}}\right|$, the phase angle at each time point $\varphi_{t}=$ $\arctan (\operatorname{Im}[\mathrm{Z}(t)] / \operatorname{Re}[\mathrm{Z}(t)]), n$ is the number of trials, $j$ is the trial, and $i$ is the complex operator.

To test the hypothesis that third-flash perception is related to more precise phase alignment of oscillatory responses to veridical flashes, we computed phase consistency across trials at $11.43 \mathrm{~Hz}$ (corresponding to the $87.5 \mathrm{~ms} \mathrm{SOA}$ ) using the weighted pairwise phase consistency (wPPC) metric (Vinck et al., 2010). Consistency of phases across trials is often estimated using ITPC. However, ITPC is sensitive to relative and absolute trial count (Cohen, 2014a), whereas wPPC corrects for this bias because it measures similarity of phase angles between all trial pairs. The formula for wPPC as implemented in Fieldtrip function $f t \_c o n n e c t i v i t y \_p p c . m$ is as follows:

$$
w P P C=\frac{\left|\sum_{j=1}^{N} Z\right|^{2}-\sum_{j=1}^{N}|Z|^{2}}{\left.\left|\sum_{j=1}^{N}\right| Z\right|^{2}-\sum_{j=1}^{N}|Z|^{2}}
$$

where $Z$ is a complex convolution result computed at each time point using 3-cycle Morlet wavelet as described above. Conceptually, wPPC measures the extent to which the circular distance between phase angle pairs taken from different trials is nonuniformly distributed, whereas ITPC measures the extent to which a distribution of phase angles across trials at each time point is nonuniform. Analytically, wPPC is comparable with ITPC ${ }^{2}$ (Vinck et al., 2010).

Statistical analyses. Differences in the prestimulus and poststimulus alpha-band power were performed taking into account IAF, and its variability across parietal and occipital alpha sources (Haegens et al., 2014). Therefore, for each participant, alpha band was defined as IAF $\pm 1.5 \mathrm{~Hz}$ separately for parietal and occipital alpha sources. Statistical comparison of prestimulus alpha-band power was performed on raw (i.e., nonbaseline-corrected power values). Statistical comparison of prestimulus ( -600 to $0 \mathrm{~ms}$ ) and poststimulus ( 0 to $600 \mathrm{~ms}$ ) alpha-band time courses, and $\mathrm{wPPC}$ between illusion and nonillusion trials was performed using nonparametric permutation testing procedure as described further (Maris and Oostenveld, 2007).

First, real $t$-values were obtained by performing a one-sample $t$-test at each time point comparing the time-series of condition difference in power (or wPPC) against 0 . Second, a null hypothesis distribution of $t$-values (permuted $t$-values) was created by randomly assigning condition labels (mathematically, this was implemented by multiplying the condition difference time-series from a random number of participants by -1$)$ and computing $t$-values. This procedure was repeated 1000 times. Third, the real $t$-values were $z$-scored using the mean and SD of permuted $t$ values. Fourth, power (or wPPC) differences were considered statistically significant if the $z$-scored $t$ value at each time point was larger than $95 \%$ of permuted $t$-values (i.e., $z$-score $>1.65$ ). Finally, cluster-based correction was applied to correct for multiple comparisons over time points. The null hypothesis distribution of cluster sizes was obtained as follows. At each of 1000 iterations of permutation testing, the permuted $t$-value time series was thresholded at $p<0.05$, and the maximum cluster size value (sum of $t$-values within a cluster) was stored. Clusters of contiguous time points in the real $t$-value time series were considered significant if the size of the cluster was bigger than expected under the null hypothesis. To obtain more stable estimates from permutation testing, 
A

Finely sampled SOAs $(\mathrm{N}=9)$

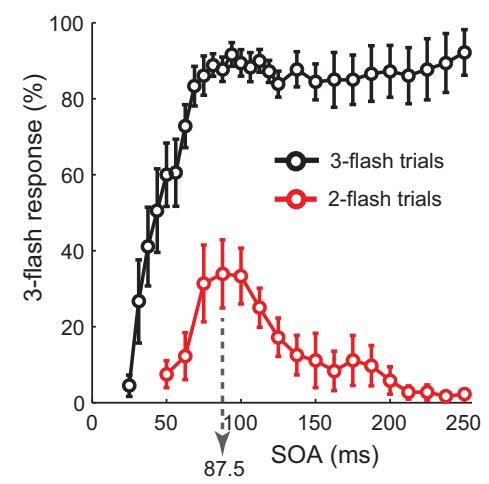

Coarsely sampled SOAs $(\mathrm{N}=18)$

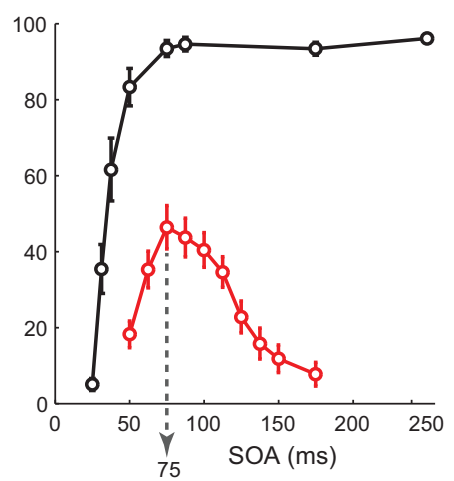

B

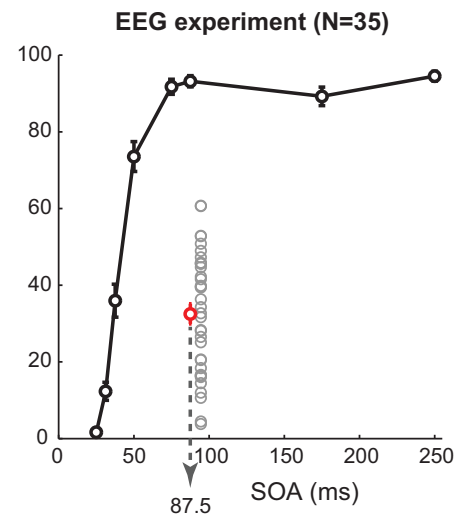

Figure 2. Behavioral performance. Percentage of three-flash percepts for two- and three-flash trials as a function of SOA between veridical flashes. $\boldsymbol{A}$, Behavioral results of psychophysics Experiment 1, where fine (17 SOAs for two- and 27 S0As for three-flash trials) and coarse (10 SOAs for the two-and 8 SOAs for the three-flash trials) were used. Dotted arrows indicate tw0-flash trial SOA that produced on average the maximum number of illusions. B, Behavioral results of the EEG experiment (Experiment 2), where the two-flash trial SOA was fixed at 87.5 ms. Gray circles represent average percentage of illusions for each participant $(N=35)$. Error bars indicate SEM.

we ran a "meta-permutation test" by repeating pixel-level and clusterlevel permutation procedure 20 times. Thus, the average of 20 real $z$-scored $t$-values and the average of 20 cluster thresholds were used.

Statistical significance of POS was tested by comparing the observed POS value at each time-frequency point to the null hypothesis distribution of POS values, which was obtained using the following procedure. For each participant, illusion and nonillusion trials were randomly relabeled and surrogate POS was computed. This was repeated 1000 times at each time-frequency tile. Thus, obtained 1000 surrogate POS values per subject were then used to compute 100,000 grand-average surrogate POS values (i.e., average across subjects). At each of 100,000 iterations, one surrogate POS value was selected for each participant and averaged across participants. Finally, $p$ value was computed as the proportion of grand-average surrogate POS values that exceeded empirically observed grand-average POS. This $p$ value indicates how empirically observed phase differences between illusion and nonillusion trials deviated from phase differences expected under null hypothesis (see Fig. 5). Statistical comparison of POS frequency profile was performed by summing the observed and surrogate grand-average POS values over time ( -600 to $0 \mathrm{~ms}$ ) and computing the $p$ value as described above. Correction for multiple comparisons across frequencies was performed using nonparametric cluster correction procedure, equivalent to that used for alpha power and $\mathrm{wPPC}$ comparison between conditions.

\section{Results}

\section{Experiment 1}

In Experiment 1, we empirically tested Bowen's theoretical idea that the triple-flash illusion occurs when the delay between veridical flashes matches the period of IRF generated in response to a single flash. The two key components of Bowen's model were determined using a psychophysical approach (subject-specific optimal delay between flashes) and EEG recordings (period of the subject-specific IRF).

\section{Evidence for Bowen's model}

Replicating Bowen's results (Bowen, 1989), we found that illusory third-flash perception depended on SOA between the two veridical flashes. The main effect of SOA on two-flash trials was significant for both coarsely $\left(N=18 ; F_{(9,153)}=20.86, p<0.001\right)$ and finely sampled SOAs $\left(N=9 ; F_{(16,128)}=4.85, p<0.001\right)$, such that perception of illusions followed an inverted $U$-shaped function (Fig. 2A). Averaged across finely and coarsely sampled equivalent SOAs, a $75 \mathrm{~ms}$ delay between two veridical flashes yielded the strongest third-flash illusion: At this SOA, the illusory third flash was perceived on approximately half of the two-flash trials $(41.41 \pm 26.83 \%)$. In contrast, three veridical flashes separated by the same $75 \mathrm{~ms}$ SOA almost always were perceived as three $(91.04 \pm 11.86 \%)$, indicating that perception of the illusory flash on two-flash trials does not result from an inability to distinguish rapidly presented stimuli. This was further supported by the main effect of SOA on three-flash trials (for finely sampled SOAs, $F_{(1,26)}=23.27, p<0.001$; for coarsely sampled SOAs, $F_{(1,7)}=$ 94.35, $p<0.001$ ), where perception of three flashes steadily increased as a function of SOA (Fig. 2A).

To directly test Bowen's theoretical account of the triple-flash illusion, we first determined each participant's IRF and its period. As previously reported (VanRullen and Macdonald, 2012), white noise stimuli that have a flat frequency spectrum can be used to reveal subject-specific IRF by cross-correlating stimulus luminance time series with concurrently recorded EEG time series (Fig. 3B). As in previous reports (İlhan and VanRullen, 2012; VanRullen and Macdonald, 2012), the cross-correlation result was oscillatory, with a period of $\sim 100 \mathrm{~ms}$ on average and maximal amplitude over occipital electrodes (Fig. 3D). Next, we determined subject-specific SOA that maximized illusory thirdflash perception by fitting symmetrical and nonsymmetrical functions (Gaussian, Weibull, and ex-Gaussian) to behavioral performance on two-flash trials. Given that ex-Gaussian function provided the best model fits $\left(M\left(R_{\text {Gaussian }}^{2}\right)=0.89, S D\left(R_{\text {Gaussian }}^{2}\right)=\right.$ $0.09 ; M\left(R_{\text {Weibull }}^{2}\right)=0.89, S D\left(R_{\text {Weibull }}^{2}\right)=0.08 ; M\left(R_{\text {ex-Gaussian }}^{2}\right)=$ $\left.0.91, S D\left(R_{\text {ex-Gaussian }}^{2}\right)=0.08\right)$, the peak of fitted ex-Gaussian function was taken as a subject-specific optimal SOA (Fig. 3A).

The correlation between two key elements of Bowen's model (subject-specific SOA that maximized illusory perception and period of subject-specific IRF) was significant $\left(r_{\text {Pearson(25) }}=0.51\right.$, $p_{\text {two-tailed }}=0.006, \mathrm{CI}_{95 \%}=[0.170 .75]$; Fig. $\left.3 C\right)$. This result is the first direct evidence for Bowen's theoretical account of the tripleflash illusion. To evaluate the influence of the outliers, we recomputed correlation for 1000 times by randomly resampling with replacement. The $95 \%$ percentile CI of these bootstrapped correlations did not include $0\left(\mathrm{CI}_{95 \%}=[0.170 .72]\right)$, indicating robustness of the observed effect. For consistency purposes with other analyses, we also tested resistance to outliers by using a nonparametric permutation testing procedure, by randomly shuffling IRF values across participants and recomputing correlation for 
A

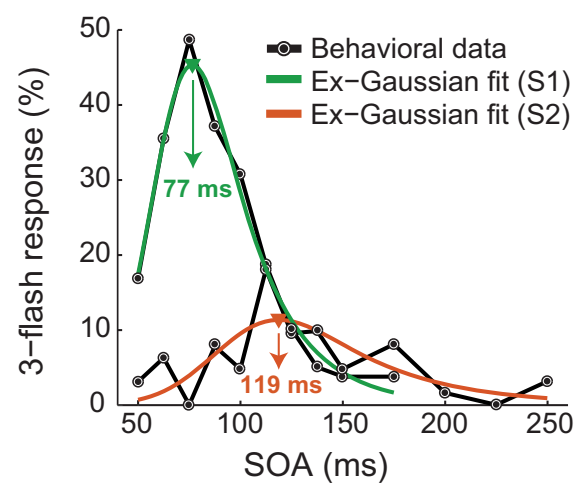

B

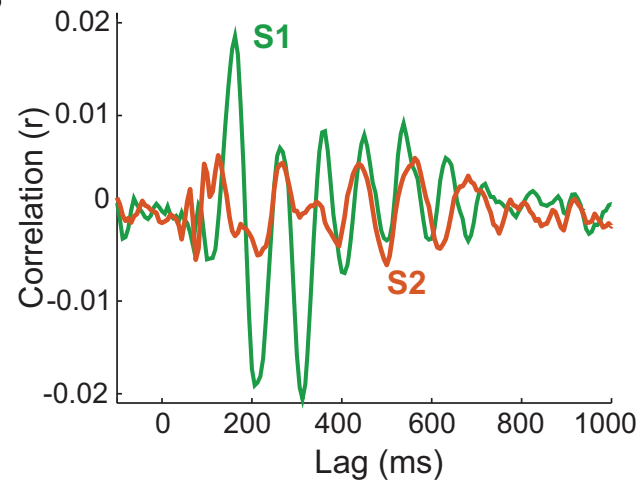

C

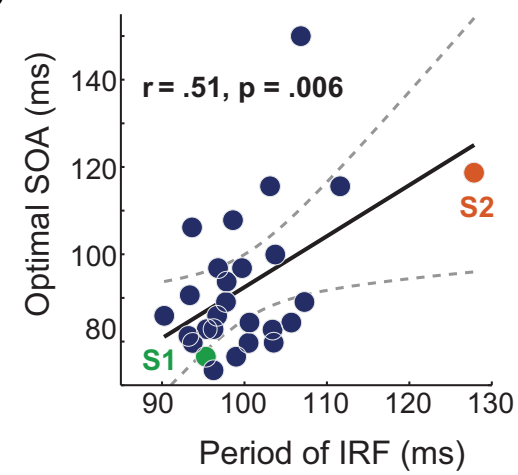

D

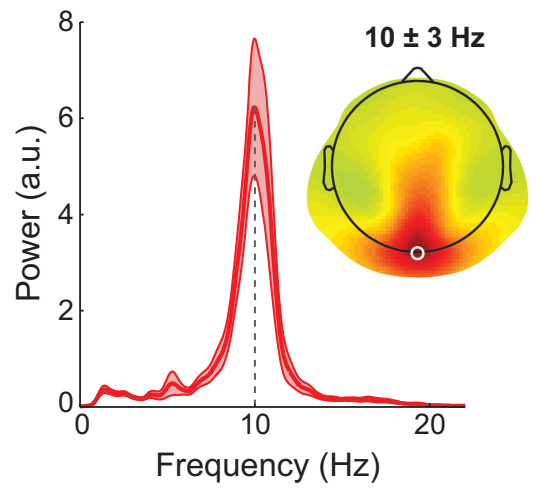

Figure 3. Relationship between period of IRF and optimal delay between flashes. $\boldsymbol{A}$, Behavioral data from 2 participants (S1 and S2) showing the probability of third-flash perception on two-flash trials as a function of SOA (black lines), and ex-Gaussian fits used to determine subject-specific optimal SOA (green and orange lines). $\boldsymbol{B}$, Single-subject IRFs at $0 z$ electrode (same participants as in A). C, Correlation (across participants, $N=27$ ) between period of IRF at $0 z$ electrode and optimal SOA. Dashed curves indicate $95 \%$ Cls around the slope of regression line. Green and orange dots represent participants' data depicted in $\boldsymbol{A}, \boldsymbol{B}$. D, Subject-average power spectrum of IRF at $0 \mathrm{z}$ and topographical map of $10 \pm 3 \mathrm{~Hz}$ power, indicating that IRF is strongest around $0 \mathrm{z}$ (white circle). Subject-average power spectrum of IRF at $0 z$ electrode with a peak centered at $10 \mathrm{~Hz}$. Light red areas represent SEM.

1000 times (Maris and Oostenveld, 2007). The empirically observed correlation was significantly different from the null hypothesis distribution $(z$-score $=2.69, p=0.0036)$, indicating robustness of the observed effect.

\section{Experiment 2}

In Experiment 2, we investigated the role of alpha-band oscillations in generation of the triple-flash illusion. For this, we conducted an EEG experiment, for which SOA on two-flash trials was fixed at $87.5 \mathrm{~ms}$ based on the results of Experiment 1, where maximal number of illusions on average across participants for ex-Gaussian fits was $92 \mathrm{~ms}(S D=17 \mathrm{~ms})$. The fixed SOA was chosen to maximize the number of illusion trials for subsequent phase-based analyses that are sensitive to the number of trials (Vinck et al., 2010; Cohen, 2014a; VanRullen, 2016a).

\section{Third-flash perception depends on individual alpha frequency}

Why did some participants perceive the illusion nearly half of the time and others perceived virtually none (Fig. $2 B$ )? Based on the previously reported correlation between IRF frequency and occipital IAF (VanRullen and Macdonald, 2012), we hypothesized that variability in IAF could be related to the observed betweensubject differences in proneness to perceive the third-flash illusion. Specifically, the closer the match between IAF and fixed SOA used in the EEG experiment $(87.5 \mathrm{~ms} \sim 11.43 \mathrm{~Hz})$, the more illusions a given participant would perceive. We focused on the frequency of task-related (as opposed to resting-state) alpha- band oscillations before the first flash because IAF has been shown to be state-dependent (Haegens et al., 2014). Furthermore, parietal and occipital IAFs were determined in the prestimulus window to avoid contamination from sensory stimulus processing that is accompanied by rapid instantaneous frequency changes in all frequency bands (Burgess, 2012). To account for IAF variability across brain regions (Klimesch, 1999; Haegens et al., 2014), we isolated occipital and parietal alpha sources using independent component analysis (for details, see Materials and Methods). For two participants, alpha-band sources could not be determined due to small alpha peaks in the power spectrum that were indistinguishable from noise. Thus, 33 participants were included in the correlation analyses. On average, there were 5.36 ICs $(S D=2.01)$ per subject with a clear alpha peak in the frequency spectrum and residual variance of the dipole fit (mismatch between the component scalp map and the scalp projection of a fitted dipolar source) of $<15 \%$. The average residual variance of the dipole fit for the selected parietal ICs was $3.77 \%(\mathrm{SD}=2.39 \%)$, and for the occipital ICs was $5.07 \%(\mathrm{SD}=3.34 \%)$.

The normalized power spectra for all participants is plotted in Figure $4 B$. Average peak frequency in parietal ROI was $10.4 \mathrm{~Hz}$ $(\mathrm{SD}=0.94)$, and in occipital ROI $-10.5 \mathrm{~Hz}(\mathrm{SD}=1.1)$. Average peak frequency of alpha oscillations across participants statistically did not differ between the two ROIs $\left(_{(32)}=-0.625, p=\right.$ 0.536).

Alpha peak frequency is not stationary (Cohen, 2014b; Samaha and Postle, 2015), and fluctuations around IAF, albeit small $(\sim 0.04 \mathrm{~Hz})$ (Samaha and Postle, 2015), can be relevant for per- 
A
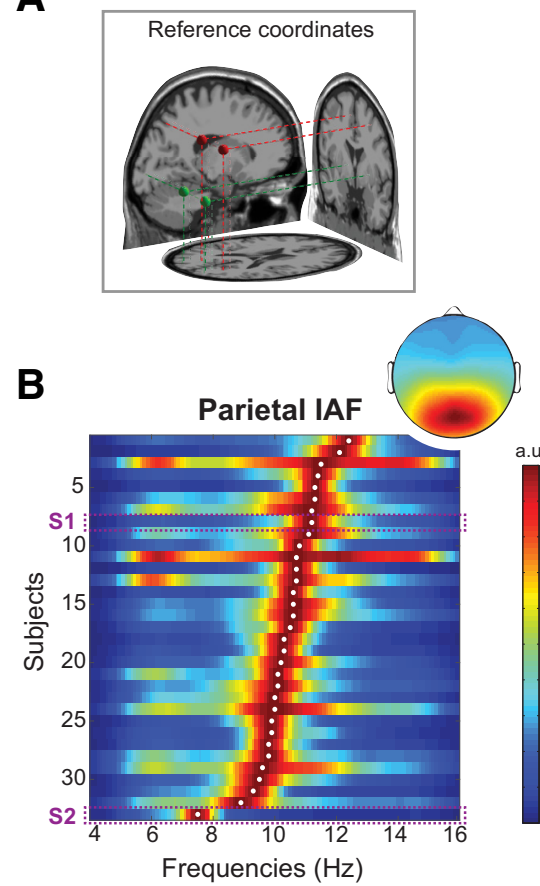

C

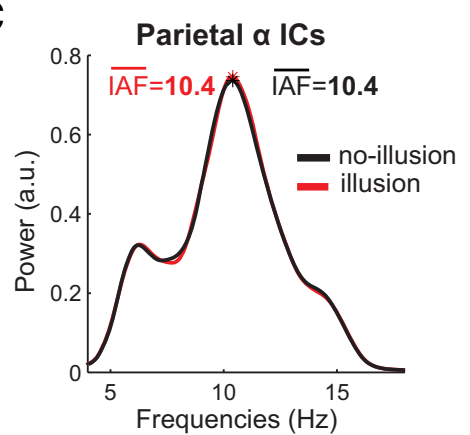

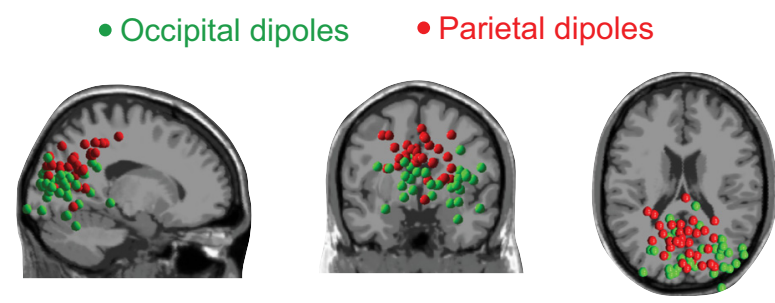

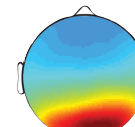

Occipital IAF

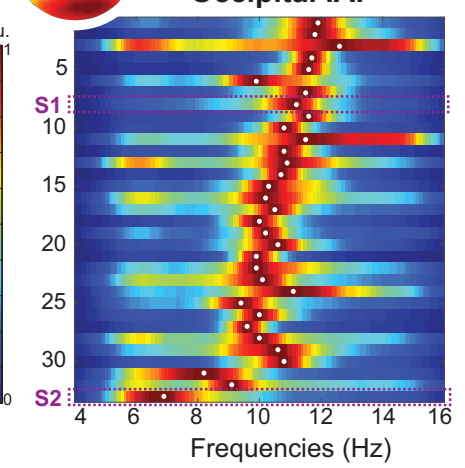

Occipital a ICs

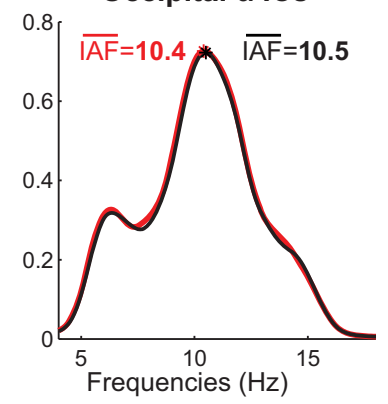

D

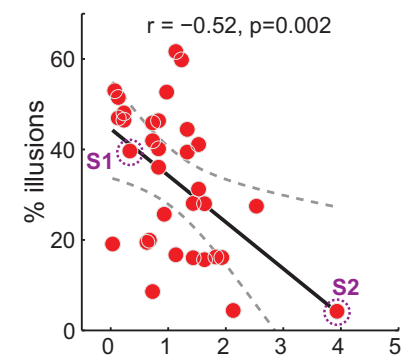

Distance from $11.43 \mathrm{~Hz}$ to IAF $(\mathrm{Hz})$

E

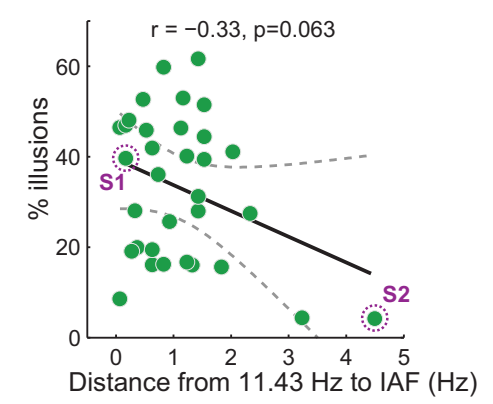

Figure 4. Relationship between the frequency of alpha oscillations and third-flash illusory percepts. $A$, Dipole locations for anatomical reference coordinates for parietal and occipital ROls (leftmost panel). Locations of equivalent dipoles for parietal and occipital alpha ICs. B, Power spectra of alpha parietal and occipital IC time series in the prestimulus window ( -1000 to 0 ms, where 0 is the first flash), normalized to the power of each participant's alpha peak (for comparability across participants). Each row represents a participant. Color represents normalized spectral power. White dot represents the individual's alpha peak frequency. Dashed rectangles represent the power spectra of exemplar Subjects $S 1$ and $S 2$ depicted in Figure $3 A, B$. Inset, Topographical maps represent scalp projections of parietal and occipital equivalent dipole centroids, revealing more central and anterior projection for parietal ICs. C, Subject-average normalized power spectra of occipital and parietal IC time series in the prestimulus window, illustrating that IAF defined from all versus separate classes of trials did not differ. $D, E$, Correlations (across participants, $N=33$ ) between IAF and overall percentage of illusions, indicating that the proportion of illusions depended more strongly on the match between interflash delay $(11.43 \mathrm{~Hz} \sim 87.5 \mathrm{~ms})$ and IAF at parietal (D) than occipital alpha sources $(\boldsymbol{E})$.

ception. To control for the possibility that IAF estimated in the prestimulus window using all trials was disproportionally influenced by one class of trials (e.g., nonillusion trials), we additionally determined IAF preceding illusion and nonillusion trials separately. The subject-average $(N=33)$ normalized power spectra for illusion and nonillusion trials separately are represented in Figure $4 C$, and show highly overlapping spectral profiles. A paired $t$ test of IAF for illusion and nonillusion trials was not significant for either parietal $\left(t_{(32)}=-0.34, p=0.735\right)$ or occipital alpha sources $\left(t_{(32)}=-0.22, p=0.827\right)$. Thus, differences in IAF before illusion versus nonillusion trials were negligible relative to IAF differences between parietal and occipital ROIs when compared across participants $(0.6 \mathrm{~Hz})$.

As hypothesized, the probability of the third-flash perception using fixed SOA was correlated with IAF: the smaller the absolute distance between parietal IAF and $11.43 \mathrm{~Hz}$, the more illusions participant perceived. The correlation was significant for the parietal alpha sources $\left(r_{\text {Pearson(31) }}=-0.52, p_{\text {two-tailed }}=0.002\right.$, $\mathrm{CI}_{95 \%}=[-0.73-0.21]$; Fig. $\left.4 C\right)$ and showed the same trend at the occipital alpha sources $\left(r_{\text {Pearson(31) }}=-0.33, p_{\text {two-tailed }}=\right.$ $0.063, \mathrm{CI}_{95 \%}=[-0.600 .02]$; Fig. $\left.4 D\right)$. Robustness of the correlations was evaluated using bootstrapping procedure, which revealed that the $95 \%$ percentile $\mathrm{CI}$ of bootstrapped correlations for

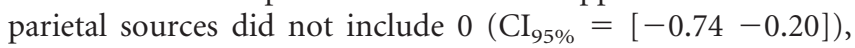
whereas for occipital sources it $\operatorname{did}\left(\mathrm{CI}_{95 \%}=\left[\begin{array}{l}-0.620 .14\end{array}\right]\right)$. A nonparametric permutation testing procedure indicated that the empirically observed correlation was significantly different from the null hypothesis distribution $(z$-score $=-2.97, p=0.0015)$ for the parietal as well as occipital IAF $(z$-score $=-1.85, p=0.03)$.

To link the findings of between-subject correlations from both Experiment 1 and Experiment 2, we correlated the peak frequency of IRF determined in Experiment 1 with IAF determined in the Experiment 2. Replicating the previous findings (VanRullen and Macdonald, 2012), the peak of IAF strongly cor- 
A

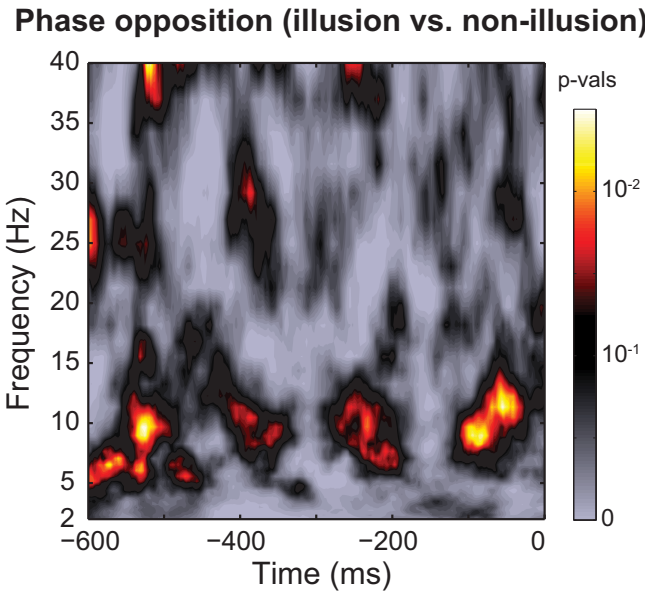

B

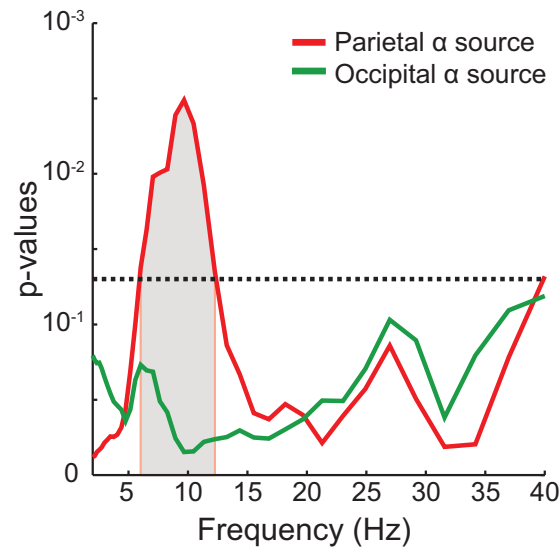

Figure 5. Prestimulus alpha phase differences between illusion and nonillusion trials. $\boldsymbol{A}$, Time-frequency representation of $p$ values (for parietal alpha sources) computed as a proportion of surrogate phase opposition values (distribution of phase opposition values expected under null hypothesis) that exceeded empirically observed phase opposition values. $\boldsymbol{B}$, Frequency profile of phase opposition (averaged over -600 to 0 ms prestimulus time window). Gray shaded area represents frequencies at which the observed phase opposition frequency profile was significantly different from the frequency profile of surrogate phase opposition values (corrected for multiple comparisons across frequencies using cluster-based permutation testing). Horizontal line indicates $p=0.05$.

related with both parietal $\left(r_{\text {Pearson(16) }}=0.818, p_{\text {two-tailed }}<0.001\right)$ and occipital IAF $\left(r_{\text {Pearson(16) }}=0.774, p_{\text {two-tailed }}<0.001\right)$. Thus, there is a close link between oscillatory responses to a luminance increment (IRF) and endogenous posterior alpha brain rhythms (IAF).

\section{Prestimulus alpha phase predicts third-flash perception}

Although correlation between subject-specific optimal SOA and the period of subject-specific IRF lends support to Bowen's notion that the triple-flash illusion reflects a superposition of two oscillatory responses, it does not explain trial-to-trial variability in perception of the illusion. At the subject-specific optimal SOA, the third flash is only perceived on average half of the time $(45 \%$ in the Experiment 2). To address this question, we contrasted brain activity during physically identical two-flash trials on which the third flash was either reported or not. All within-subject analyses were performed on two alpha component time series (occipital and parietal), which were determined based on spectral and spatial characteristics (for details, see Materials and Methods).

We examined the effects of prestimulus alpha phase on perceptual outcome by computing the POS, a measure that represents the extent to which phase distributions between two classes of trials differ (Busch et al., 2009; VanRullen, 2016a) (here, illusion and nonillusion trials). Given that statistical power of POS is influenced by the absolute trial count in each class of trials (VanRullen, 2016a), and that reliability of POS can be compromised in case of unequal illusion and nonillusion trial counts (an inherent feature of all phase-based time-frequency analyses methods) (Vinck et al., 2010; Cohen, 2014a), we selected only participants for which illusion and nonillusion trial counts differed by $<10 \%$ $(N=14)$. The average number of illusion trials was 333 (SD = $43)$, and nonillusion trials was $399(\mathrm{SD}=33)$.

If the phase of spontaneous alpha oscillations before the first flash is predictive of the third-flash perception, we should observe a strong phase clustering around a certain phase angle for illusion trials accompanied by strong phase clustering around the opposite phase angle for nonillusion trials. Prestimulus alpha phase differed at parietal, but not occipital, alpha sources (Fig. 5). The POS spectrum (averaged across all time points in the prestimulus interval) at parietal alpha sources was statistically significant in
6-12 Hz frequency range (corrected for multiple comparisons across frequencies using cluster-based permutation testing). Although analyses were performed on alpha sources, finding phaseopposition in the alpha band is not trivial, as source-separation was not based on phase measures.

Next, we tested the effects of prestimulus alpha power (Fig. 6). Within-subject trial-by-trial fluctuations of prestimulus alpha power have been shown to affect both near-threshold stimulus perception as well as perception of illusions (Romei et al., 2008; Lange et al., 2014). Moreover, some studies reported that between-subject differences in occipital alpha power are correlated with proneness for illusory perception (Cecere et al., 2015) and performance in the perceptual discrimination tasks (Hanslmayr et al., 2007; Limbach and Corballis, 2016).

Within-subject alpha power analysis revealed significantly lower prestimulus alpha power on illusion versus nonillusion trials at parietal (all $p$ values $<0.05$ in the time interval -520 to $-270 \mathrm{~ms}$ relative to first flash, corrected for multiple comparisons using cluster-based permutation testing), but not occipital alpha sources (all $p$ values $>0.05$ in the time interval -600 to 0 $\mathrm{ms})$. For between-subject alpha power analyses $(N=33)$, we computed subject-specific alpha power averaged across illusion and nonillusion trials in the prestimulus window $(-500$ to -300 ms) using two frequency bands: (1) around IAF $\pm 1.5 \mathrm{~Hz}$ (for direct comparison with within-subject analyses) and (2) at the stimulus presentation frequency $11.43 \pm 1.5 \mathrm{~Hz}$. Correlations between prestimulus alpha power (around IAF) and overall percentage of illusions was not significant for either parietal $\left(r_{\text {Pearson(31) }}=-0.15\right.$, $\left.p_{\text {two-tailed }}=0.397\right)$ or for occipital alpha sources $\left(r_{\text {Pearson(31) }}=\right.$ $\left.0.16, p_{\text {two-tailed }}=0.381\right)$. The correlation results were also nonsignificant when defining alpha power around the stimulation frequency (i.e., $11.43 \pm 1.5 \mathrm{~Hz}$ ): The correlation at parietal alpha sources was $r_{(31)}=-0.028\left(p_{\text {two-tailed }}=0.879\right)$, and occipital alpha sources was $r_{(31)}=0.195\left(p_{\text {two-tailed }}=0.276\right)$.

To rule out that IAF estimation was affected by betweensubject differences in alpha power, we also correlated IAF with absolute prestimulus alpha power. None of the correlations was significant: The correlation with parietal alpha power around IAF was $r_{\text {Pearson(31) }}=0.01\left(p_{\text {two-tailed }}=0.945\right)$, and around 11.43 was $r_{\text {Pearson(31) }}=0.20\left(p_{\text {two-tailed }}=0.266\right)$; the correlation with occip- 


\section{A}

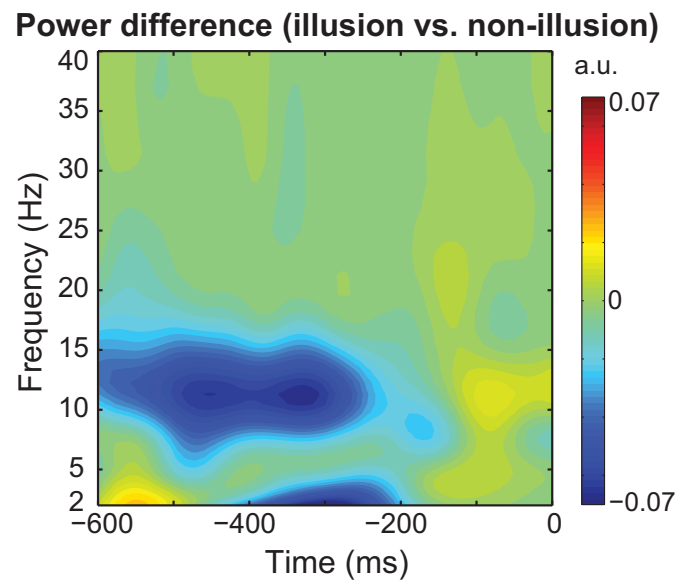

B

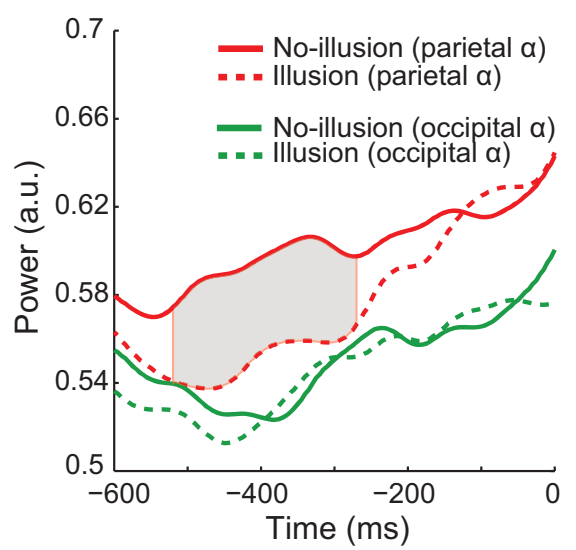

Figure 6. Prestimulus alpha power differences between illusion and nonillusion trials. $\boldsymbol{A}$, Time-frequency representation of alpha power differences at parietal sources. $\boldsymbol{B}$, Time courses of alpha power (IAF $\pm 1.5 \mathrm{~Hz}$ ) for illusion (dashed lines) and nonillusion (solid lines) trials at occipital and parietal alpha sources, demonstrating that alpha power differences were present at parietal, but not occipital, alpha sources. Gray shaded area represents the time interval where statistically significant differences between the two trial groups was observed (corrected for multiple comparisons using cluster-based permutation testing).
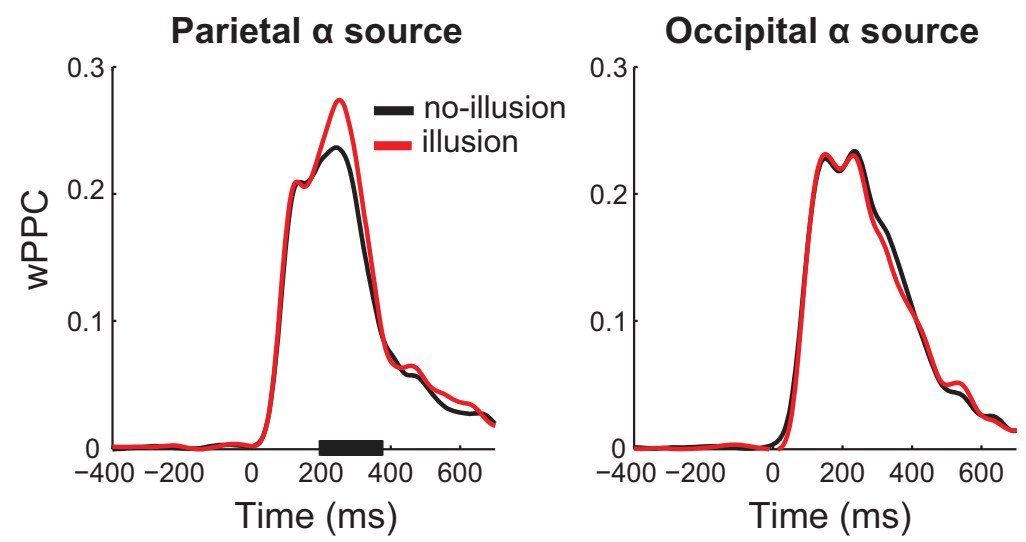

Figure 7. Pairwise phase consistency for illusion and nonillusion trials. Time courses of wPPC at $11.43 \mathrm{~Hz}$ for illusion and nonillusion trials plotted separately for parietal (left) and occipital (right) alpha sources. Black bar on the $x$-axis represents time points at which wPPC for illusion versus nonillusion trials was significantly different (corrected for multiple comparisons using cluster-based permutation testing).

ital alpha power around IAF was $r_{\text {Pearson(31) }}=0.21\left(p_{\text {two-tailed }}=\right.$ 0.243 ), and around 11.43 was $r_{\text {Pearson(31) }}=0.27$ ( $p_{\text {two-tailed }}=$ $0.127)$. Together, these results demonstrate that trial-by-trial alpha power fluctuations (rather than between-subject differences in alpha power) at parietal, but not occipital, alpha sources are one of the elements determining the probability to perceive an illusion on a given trial.

\section{Illusory perception is associated with stronger poststimulus local phase alignment}

According to Bowen's theoretical model, presentation of the second flash in-phase with the oscillatory IRF evoked by the first flash would result in perfect superposition of the two IRFs and hence the perception of an illusory third flash. Whenever, for any reason (e.g., nonoptimal SOA, nonoptimal alpha phase at the first-flash onset, variability in stimulus-evoked oscillatory alpha phase), the second flash does not occur in-phase with the oscillatory response evoked by the first flash, then the model stipulates that third-flash perception would be less likely. Thus, we predicted more precise phase alignment on illusion than nonillusion trials in the poststimulus window. We assessed phase alignment by computing wPPC metric (Vinck et al., 2010) at the frequency of the two veridical flashes $(11.43 \mathrm{~Hz} \sim 87.5 \mathrm{~ms})$. As illustrated in Figure 7, we found significantly higher wPPC for illusion than for nonillusion trials at parietal, but not occipital, alpha sources, indicating higher phase consistency. We also compared poststimulus alpha power to rule out the possibility that wPPC differences were a result of less accurate phase estimation due to low alpha power (Cohen, 2014a). We observed a typical decrease in alpha power related to stimulus processing, but this effect did not differ between illusion and nonillusion trials.

\section{Discussion}

We validated and extended the original theoretical account of the triple-flash illusion proposed by Bowen (1989), according to which the illusory third-flash percept arises when the delay between the two veridical flashes matches the period of a hypothetical oscillatory IRF generated in response to each stimulus. In Experiment 1, we demonstrated that the subject-specific interflash delay for which the illusory perception is maximized was strongly correlated with the period of the oscillatory IRF, which reverberates at $\sim 10 \mathrm{~Hz}$ (Fig. 3C). The subject-specific IRF was derived from EEG responses to white-noise luminance sequences by crosscorrelating the two signals (VanRullen and Macdonald, 2012). In Experiment 2, when fixing the interflash delay $(87.5 \mathrm{~ms})$ for all participants, we demonstrated that IAF of parietal compared with occipital alpha sources was more strongly correlated with the overall proportion of illusory percepts: The closer participant's parietal alpha peak was to $11.43 \mathrm{~Hz}$ (the $87.5 \mathrm{~ms}$ delay, expressed in $\mathrm{Hz}$ ), the more illusions were perceived. Together, these results point to an active or "driving" (as opposed to modulatory) role of alpha-band oscillations in perception, and reveal that alpha-band reverberations to a single stimulus have direct consequences on perception spanning several subsequent alpha 
cycles. Moreover, these findings emphasize the importance of using the "individual differences" approach when studying perceptual cycles and their associated oscillatory signatures.

Parietal and occipital alpha sources were estimated from ICA and single dipole fitting: a combination of methods that appropriately dissociates highly spatially adjacent oscillatory sources (Töllner et al., 2017). Although ICs are often dipolar (Delorme et al., 2012), the anatomical dissociation of occipital versus parietal sources should be interpreted cautiously, considering that dipole localization was based on 64-channel EEG using standard electrode locations, and a standard anatomical head model (although standard head models can provide reasonably high localization accuracy) (Fuchs et al., 2002). However, a differential role of occipital versus parietal alpha in perception has been reported previously and is consistent with our findings. In a discrimination task, for example, lower alpha power in parietal sources (BA 7) preceded correct trials and was interpreted to reflect information gating from occipital to dorsal parietal areas controlled by top-down effects of attention (van Dijk et al., 2008). Importantly, this parietal alpha source was distinct from the occipital alpha source identified from the resting-state recordings. Relatedly, behavioral effects of $10 \mathrm{~Hz}$ rTMS in another visual discrimination task were observed only when stimulating parietal, but not occipital, areas (Jaegle and Ro, 2014). Although this effect might be due to stronger entrainment effects in parietal compared with occipital rTMS, the proposed active role of parietal alpha in modulating visual representations in lower visual areas could account for the differential rTMS effects (Foxe and Snyder, 2011; Palva and Palva, 2011; Kwon et al., 2017). Prestimulus alpha phase in frontoparietal areas has also been demonstrated to affect the connectivity between occipital and parietal areas, with certain prestimulus alpha phases associated with increased connectivity and better nearthreshold stimulus detection (Hanslmayr et al., 2013).

Only two empirical studies of the triple-flash illusion, to our knowledge, have been conducted since Bowen's original report; they compared the optimal SOA of the triple-flash illusion between healthy controls and schizophrenia patients (Norton et al., 2008; Chen et al., 2014). In both studies, the average SOA that maximized illusory perception was longer in the schizophrenia patient group (130-150 ms) than in the control group (90-110 $\mathrm{ms}$ ). Following Bowen's model, the authors speculated that such differences could result from temporal dilation of the IRF in schizophrenia patients. Considering both our between-subject correlation analyses results (Figs. 3, 4) and previous reports of slower IAF in patients with chronic schizophrenia and schizophrenia symptoms (Cañive et al., 1998; Harris et al., 2006), a slowing down of occipitoparietal alpha rhythms seems a conceivable explanation for the findings of Norton et al. (2008) and Chen et al. (2014).

Although Bowen's theoretical model explains the mechanics behind the triple-flash illusion (Fig. 1B), it does not explain why at the subject-specific optimal SOA, an illusory third-flash is only perceived half of the time ( $45 \%$ on average). We hypothesized that this probabilistic nature of the illusion could be related to moment-to-moment fluctuations in occipitoparietal alpha phase and power that are known to be perceptually relevant (VanRullen et al., 2011; Kleinschmidt et al., 2012). In the EEG experiment (Experiment 2), we found that trial-to-trial variability in perception of the illusion was indeed related to the prestimulus alpha phase at parietal, but not occipital, alpha sources, such that illusion and nonillusion trials were associated with opposite alpha phases (Fig. 5). Illusion trials were also preceded by significantly lower prestimulus alpha-band power at parietal alpha sources
(Fig. 6). These findings are in accordance with previous reports linking relatively lower occipitoparietal alpha power and certain alpha phases to higher cortical excitability (Romei et al., 2008; Mathewson et al., 2009; Dugué et al., 2011; Lange et al., 2013). Variations in occipitoparietal alpha power are related not only to veridical but also illusory perception: Illusory percepts are more frequent when occipitoparietal alpha power is low, both within and between subjects (VanRullen et al., 2006; Lange et al., 2014; Cecere et al., 2015).

Presentation of stimuli in phase with endogenous alpha oscillations results in stronger phase consistency across trials compared with jittered stimulation (Thut et al., 2011; Spaak et al., 2014; Notbohm et al., 2016). In the poststimulus period, we found higher phase consistency (as measured by wPPC) for illusion than nonillusion trials at $11.43 \mathrm{~Hz}$ (the two-flash trial SOA of $87.5 \mathrm{~ms}$, translated into $\mathrm{Hz}$ ) for parietal, but not occipital, alpha sources (Fig. 7). The wPPC differences between illusion and nonillusion trials, as well as prestimulus phase-opposition effects, appear complementary with Bowen's theoretical model, which posits that illusory third-flash perception is associated with an enhancement of response amplitude resulting from phase-aligned oscillatory responses to each stimulus. Specifically, when the first stimulus appears in phase with ongoing prestimulus alpha oscillations (i.e., at the "good" prestimulus phase), there is no or relatively little phase realignment (Fellinger et al., 2011; Gruber et al., 2014); the second stimulus thus also arrives in phase with the ongoing oscillations and in phase with the oscillatory response generated to the first stimulus, resulting in high response amplitude and a third-flash percept. However, when the first stimulus arrives slightly or completely out of phase with the ongoing alpha oscillations (i.e., at the "bad" prestimulus phase), the resulting phase alignment in response to both stimuli is less precise (weaker wPPC), and thus only two flashes are perceived.

We demonstrate that perception (in a broad sense) is not only influenced by spontaneous prestimulus alpha oscillations (Busch et al., 2009; Iemi et al., 2017), but that oscillatory events related to stimulus processing on one cycle have downstream perceptual effects on multiple subsequent alpha cycles. Importantly, these nonlinear perceptual effects are most pronounced when the lag between two successive stimuli matches the rhythm of taskrelated alpha-band oscillations. This is akin to the process of entrainment of brain oscillations to rhythmic external stimuli (Thut et al., 2011), which is most effective when periodic light flashes are "in-sync" with IAF (Adrian and Matthews, 1934; Notbohm et al., 2016).

Current theoretical frameworks on the functional role of alpha oscillations posit that alpha oscillations influence sensory and cognitive processes in a pulsed manner (Klimesch et al., 2007; Jensen and Mazaheri, 2010; Mathewson et al., 2011), with strong alpha oscillations reflecting physiological inhibition, which is more pronounced at certain phases of the alpha cycle (Sadaghiani and Kleinschmidt, 2016). In the context of perceptual tasks (detection and discrimination), these theories emphasize the modulatory role of the alpha rhythm in signal processing. Consistent with the idea that the alpha rhythm represents "pulsed inhibition" (Mathewson et al., 2011), we found that illusory perception was associated with low alpha power (Fig. 6), and with a specific alpha phase (Fig. 5). However, our findings of nonlinear perceptual effects (triple-flash illusion), which result from the interaction between the subject-specific endogenous alpha rhythm and the stimulus rhythm, cannot be explained when considering alpha oscillations only as a modulatory rhythm. Instead, these results point to the driving power of alpha-band oscillations, per- 
ception of illusory stimuli without corresponding sensory input in the same or different modality, which had not been experimentally demonstrated before.

In conclusion, using the triple-flash illusion, a third illusory flash perception when only two veridical ones are presented, separated by $\sim 100 \mathrm{~ms}$, we demonstrate that alpha-band oscillations not only modulate perception but have a driving impact, which can make one perceive something that is not there.

\section{References}

Adrian ED, Matthews BH (1934) The Berger rhythm: potential changes from occipital lobes in man. Brain 57:355-385. CrossRef Medline

Apthorp D, Alais D, Boenke LT (2013) Flash illusions induced by visual, auditory, and audiovisual stimuli. J Vis 13:3. CrossRef Medline

Baumgarten TJ, Schnitzler A, Lange J (2015) Beta oscillations define discrete perceptual cycles in the somatosensory domain. Proc Natl Acad Sci U S A 112:12187-12192. CrossRef Medline

Bazanova OM, Vernon D (2014) Interpreting EEG alpha activity. Neurosci Biobehav Rev 44:94-110. CrossRef Medline

Bowen RW (1989) Two pulses seen as three flashes: a superposition analysis. Vision Res 29:409-417. CrossRef Medline

Brüers S, VanRullen R (2017) At what latency does the phase of brain oscillations influence perception? eNeuro 4:ENEURO.0078-17.2017. CrossRef Medline

Burgess AP (2012) Towards a unified understanding of event-related changes in the EEG: the firefly model of synchronization through crossfrequency phase modulation. PLoS One 7:e45630. CrossRef Medline

Busch NA, VanRullen R (2010) Spontaneous EEG oscillations reveal periodic sampling of visual attention. Proc Natl Acad Sci U S A 107:1604816053. CrossRef Medline

Busch NA, Dubois J, VanRullen R (2009) The phase of ongoing EEG oscillations predicts visual perception. J Neurosci 29:7869-7876. CrossRef Medline

Cañive JM, Lewine JD, Edgar JC, Davis JT, Miller GA, Torres F, Tuason VB (1998) Spontaneous brain magnetic activity in schizophrenia patients treated with aripiprazole. Psychopharmacol Bull 34:101-105. Medline

Cecere R, Rees G, Romei V (2015) Individual differences in alpha frequency drive crossmodal illusory perception. Curr Biol 25:231-235. CrossRef Medline

Chen Y, Norton D, Stromeyer C 3rd (2014) Prolonged temporal interaction for peripheral visual processing in schizophrenia: evidence from a threeflash illusion. Schizophr Res 156:190-196. CrossRef Medline

Cohen MX (2014a) Analyzing neural time series data: theory and practice. Cambridge, MA: Massachusetts Institute of Technology.

Cohen MX (2014b) Fluctuations in oscillation frequency control spike timing and coordinate neural networks. J Neurosci 34:8988-8998. CrossRef Medline

Delorme A, Makeig S (2004) EEGLAB: an open source toolbox for analysis of single-trial EEG dynamics including independent component analysis. J Neurosci Methods 134:9-21. CrossRef Medline

Delorme A, Palmer J, Onton J, Oostenveld R, Makeig S (2012) Independent EEG sources are dipolar. PLoS One 7:e30135. CrossRef Medline

Doppelmayr M, Klimesch W, Pachinger T, Ripper B (1998) Individual differences in brain dynamics: important implications for the calculation of event-related band power. Biol Cybern 79:49-57. CrossRef Medline

Dugué L, Marque P, VanRullen R (2011) The phase of ongoing oscillations mediates the causal relation between brain excitation and visual perception. J Neurosci 31:11889-11893. CrossRef Medline

Fellinger R, Klimesch W, Gruber W, Freunberger R, Doppelmayr M (2011) Pre-stimulus alpha phase-alignment predicts P1-amplitude. Brain Res Bull 85:417-423. CrossRef Medline

Fiebelkorn IC, Snyder AC, Mercier MR, Butler JS, Molholm S, Foxe JJ (2013) Cortical cross-frequency coupling predicts perceptual outcomes. Neuroimage 69:126-137. CrossRef Medline

Foxe JJ, Snyder AC (2011) The role of alpha-band brain oscillations as a sensory suppression mechanism during selective attention. Front Psychol 2:154. CrossRef Medline

Fuchs M, Kastner J, Wagner M, Hawes S, Ebersole JS (2002) A standardized boundary element method volume conductor model. Clin Neurophysiol 113:702-712. CrossRef Medline

Gruber WR, Zauner A, Lechinger J, Schabus M, Kutil R, Klimesch W (2014)
Alpha phase, temporal attention, and the generation of early event related potentials. Neuroimage 103:119-129. CrossRef Medline

Haegens S, Cousijn H, Wallis G, Harrison PJ, Nobre AC (2014) Inter- and intra-individual variability in alpha peak frequency. Neuroimage 92:4655. CrossRef Medline

Hanslmayr S, Aslan A, Staudigl T, Klimesch W, Herrmann CS, Bäuml KH (2007) Prestimulus oscillations predict visual perception performance between and within subjects. Neuroimage 37:1465-1473. CrossRef Medline

Hanslmayr S, Volberg G, Wimber M, Dalal SS, Greenlee MW (2013) Prestimulus oscillatory phase at $7 \mathrm{~Hz}$ gates cortical information flow and visual perception. Curr Biol 23:2273-2278. CrossRef Medline

Harris A, Melkonian D, Williams L, Gordon E (2006) Dynamic spectral analysis findings in first episode and chronic schizophrenia. Int J Neurosci 116:223-246. CrossRef Medline

Iemi L, Chaumon M, Crouzet SM, Busch NA (2017) Spontaneous neural oscillations bias perception by modulating baseline excitability. J Neurosci 37:807-819. CrossRef Medline

Illhan B, VanRullen R (2012) No counterpart of visual perceptual echoes in the auditory system. PLoS One 7:e49287. CrossRef Medline

Jaegle A, Ro T (2014) Direct control of visual perception with phase-specific modulation of posterior parietal cortex. J Cogn Neurosci 26:422-432. CrossRef Medline

Jansen BH, Brandt ME (1991) The effect of the phase of prestimulus alpha activity on the averaged visual evoked response. Electroencephalogr Clin Neurophysiol 80:241-250. CrossRef Medline

Jensen O, Mazaheri A (2010) Shaping functional architecture by oscillatory alpha activity: gating by inhibition. Front Hum Neurosci 4:186. CrossRef Medline

Kleinschmidt A, Sterzer P, Rees G (2012) Variability of perceptual multistability: from brain state to individual trait. Philos Trans R Soc Lond B Biol Sci 367:988-1000. CrossRef Medline

Klimesch W (1999) EEG alpha and theta oscillations reflect cognitive and memory performance: a review and analysis. Brain Res Brain Res Rev 29:169-195. CrossRef Medline

Klimesch W, Sauseng P, Hanslmayr S (2007) EEG alpha oscillations: the inhibition-timing hypothesis. Brain Res Rev 53:63-88. CrossRef Medline

Kwon S, Watanabe M, Fischer E, Bartels A (2017) Attention reorganizes connectivity across networks in a frequency specific manner. Neuroimage 144:217-226. CrossRef Medline

Lange J, Oostenveld R, Fries P (2013) Reduced occipital alpha power indexes enhanced excitability rather than improved visual perception. J Neurosci 33:3212-3220. CrossRef Medline

Lange J, Keil J, Schnitzler A, van Dijk H, Weisz N (2014) The role of alpha oscillations for illusory perception. Behav Brain Res 271:294-301. CrossRef Medline

Limbach K, Corballis PM (2016) Prestimulus alpha power influences response criterion in a detection task. Psychophysiology 53:1154-1164. CrossRef Medline

Maris E, Oostenveld R (2007) Nonparametric statistical testing of EEG- and MEG-data. J Neurosci Methods 164:177-190. CrossRef Medline

Mathewson KE, Gratton G, Fabiani M, Beck DM, Ro T (2009) To see or not to see: prestimulus alpha phase predicts visual awareness. J Neurosci 29: 2725-2732. CrossRef Medline

Mathewson KE, Lleras A, Beck DM, Fabiani M, Ro T, Gratton G (2011) Pulsed out of awareness: EEG alpha oscillations represent a pulsedinhibition of ongoing cortical processing. Front Psychol 2:99. CrossRef Medline

Norton D, Ongur D, Stromeyer C 3rd, Chen Y (2008) Altered 'three-flash' illusion in response to two light pulses in schizophrenia. Schizophr Res 103:275-282. CrossRef Medline

Notbohm A, Kurths J, Herrmann CS (2016) Modification of brain oscillations via rhythmic light stimulation provides evidence for entrainment but not for superposition of event-related responses. Front Hum Neurosci 10:10. CrossRef Medline

Oostenveld R, Oostendorp TF (2002) Validating the boundary element method for forward and inverse EEG computations in the presence of a hole in the skull. Hum Brain Mapp 17:179-192. CrossRef Medline

Oostenvelt R, Delorme A, Makeig S (2003) DIPFIT: equivalent dipole source localization of independent components.

Palva S, Palva JM (2011) Functional roles of alpha-band phase synchronization in local and large-scale cortical networks. Front Psychol 2:204. CrossRef Medline 
Pernet CR, Wilcox R, Rousselet GA (2012) Robust correlation analyses: false positive and power validation using a new open source matlab toolbox. Front Psychol 3:606. CrossRef Medline

Remond A, Lesevre N (1967) Variations in average visual evoked potential as a function of the alpha rhythm phase ("autostimulation"). Electroencephalogr Clin Neurophysiol 1967 [Suppl 26]:42-52. Medline

Roberts DM, Fedota JR, Buzzell GA, Parasuraman R, McDonald CG (2014) Prestimulus oscillations in the alpha band of the EEG are modulated by the difficulty of feature discrimination and predict activation of a sensory discrimination process. J Cogn Neurosci 26:1615-1628. CrossRef Medline

Romei V, Brodbeck V, Michel C, Amedi A, Pascual-Leone A, Thut G (2008) Spontaneous fluctuations in posterior alpha-band EEG activity reflect variability in excitability of human visual areas. Cereb Cortex 18:20102018. CrossRef Medline

Sadaghiani S, Kleinschmidt A (2016) Brain networks and alpha-oscillations: structural and functional foundations of cognitive control. Trends Cogn Sci 20:805-817. CrossRef Medline

Samaha J, Postle BR (2015) The speed of alpha-band oscillations predicts the temporal resolution of visual perception. Curr Biol 25:2985-2990. CrossRef Medline

Spaak E, de Lange FP, Jensen O (2014) Local entrainment of alpha oscillations by visual stimuli causes cyclic modulation of perception. J Neurosci 34:3536-3544. CrossRef Medline

Thut G, Schyns PG, Gross J (2011) Entrainment of perceptually relevant brain oscillations by non-invasive rhythmic stimulation of the human brain. Front Psychol 2:170. CrossRef Medline

Töllner T, Wang Y, Makeig S, Müller HJ, Jung TP, Gramann K (2017) Two independent frontal midline theta oscillations during conflict detection and adaptation in a Simon-type manual reaching task. J Neurosci 37: 2504-2515. CrossRef Medline

van Dijk H, Schoffelen JM, Oostenveld R, Jensen O (2008) Prestimulus oscillatory activity in the alpha band predicts visual discrimination ability. J Neurosci 28:1816-1823. CrossRef Medline

VanRullen R (2016a) How to evaluate phase differences between trial groups in ongoing electrophysiological signals. Front Neurosci 10:426. CrossRef Medline

VanRullen R (2016b) Perceptual cycles. Trends Cogn Sci 20:723-735. CrossRef Medline

VanRullen R, Macdonald JS (2012) Perceptual echoes at $10 \mathrm{~Hz}$ in the human brain. Curr Biol 22:995-999. CrossRef Medline

VanRullen R, Reddy L, Koch C (2006) The continuous wagon wheel illusion is associated with changes in electroencephalogram power at approximately 13 Hz. J Neurosci 26:502-507. CrossRef Medline

VanRullen R, Busch NA, Drewes J, Dubois J (2011) Ongoing EEG phase as a trial-by-trial predictor of perceptual and attentional variability. Front Psychol 2:60. CrossRef Medline

Vinck M, van Wingerden M, Womelsdorf T, Fries P, Pennartz CM (2010) The pairwise phase consistency: a bias-free measure of rhythmic neuronal synchronization. Neuroimage 51:112-122. CrossRef Medline 